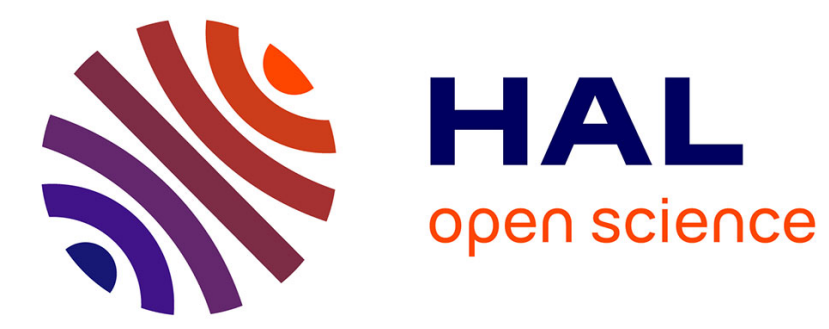

\title{
A Dual Reconfigurable 4-rRUU Parallel Manipulator
}

\author{
Abhilash Nayak, Stéphane Caro, Philippe Wenger
}

\section{To cite this version:}

Abhilash Nayak, Stéphane Caro, Philippe Wenger. A Dual Reconfigurable 4-rRUU Parallel Manipulator. 4th IEEE/IFToMM International conference on Reconfigurable Mechanisms and Robots (ReMAR2018), Jun 2018, Delft, Netherlands. 10.1109/REMAR.2018.8449902 . hal-01757553

\section{HAL Id: hal-01757553 \\ https://hal.science/hal-01757553}

Submitted on 3 Apr 2018

HAL is a multi-disciplinary open access archive for the deposit and dissemination of scientific research documents, whether they are published or not. The documents may come from teaching and research institutions in France or abroad, or from public or private research centers.
L'archive ouverte pluridisciplinaire HAL, est destinée au dépôt et à la diffusion de documents scientifiques de niveau recherche, publiés ou non, émanant des établissements d'enseignement et de recherche français ou étrangers, des laboratoires publics ou privés. 


\title{
A Dual Reconfigurable 4-rRUU Parallel Manipulator
}

\author{
Abhilash Nayak ${ }^{1}$, Stéphane Caro ${ }^{2}$ and Philippe Wenger ${ }^{2}$
}

\begin{abstract}
The aim of this paper is to introduce the use of a double Hooke's joint linkage to reconfigure the base revolute joints of a 4-RUU parallel manipulator (PM). It leads to an architecturally reconfigurable 4-rRUU PM whose platform motion depends on the angle between the driving and driven shafts of the double-Hooke's joint linkage in each limb. Even when the angle is fixed, the manipulator is reconfigurable by virtue of the different operation modes it can exhibit. By keeping the angle as a variable, the constraint equations of the 4-rRUU PM are derived using Study's kinematic mapping. Subsequently, the ideal of constraint polynomials is decomposed as an intersection of primary ideals to determine the operation modes of the 4-rRUU PM for intersecting and parallel revolute joint axes in the base and the moving platform.
\end{abstract}

\section{INTRODUCTION}

Reconfigurability in a PM extends its employability for a variety of applications. A lower mobility PM with $d o f<6$ is reconfigurable if it has different configuration space regions with possibly different type or number of degrees of freedom. These regions are known as the operation modes of the PM and were first exemplified by Zlatanov et al. [1] for the 3-URU DYMO robot exhibiting five different types of platform motion. Its closely related SNU 3-UPU [2] and Tsai 3-UPU [3] PMs were analyzed by Walter et al. with a complete characterization of their operation modes along with the transition poses. Most of the operation modes of the 3-URU or the 3-UPU PMs are physically distinguishable unlike the 3-[PP]S PMs for which the first two joints in each limb generate a motion equivalent to two coplanar translations followed by a spherical joint. 3-RPS [4], 3-PRS [5] and 3-SPR PMs [6] are such manipulators that exhibit two operation modes each with coupled motion. Other reconfigurable PMs include the 3-RER PM (E denotes a planar joint) found to have 15 3-dof operation modes [7] and the 4-RUU PM with vertical base and platform revolute joint axes possessing three operation modes [8].

Besides, a PM can be reconfigurable also by changing the position and/or orientation of one or more of its constituent joints. This type of reconfigurability is named architectural reconfigurability in this paper. MaPaMan [9] is one such manipulator in which the strut can be oriented to have two different architectures of the same PM where it can transition between roll-pitch-heave and roll-pitch-yaw degrees of freedom. Gan et al. [10] introduced a novel reconfigurable

\footnotetext{
${ }^{1}$ Abhilash Nayak is with École Centrale de Nantes, Laboratoire des Sciences du Numérique de Nantes (LS2N), 1 rue de la Noë, UMR CNRS 6004, 44321 Nantes, France abhilash. nayakels2n.fr

2 Stéphane Caro and Philippe Wenger are with CNRS, Laboratoire des Sciences du Numérique de Nantes (LS2N), École Centrale de Nantes, 1 rue de la Noë, UMR CNRS 6004, 44321 Nantes, France \{stephane.caro, philippe.wenger\}els 2 n.fr
}

revolute joint and proposed a metamorphic 3-rRPS PM. In this paper, a double Hooke's joint linkage is used as a reconfigurable revolute joint and is used to demonstrate different types of reconfigurability of a 4-RUU PM.

The double Hooke's joint linkage is a well-known special over-constrained 6R-mechanism, where the first three and the last three joint axes are mutually perpendicular. It is also known as a Double Cardan Joint and is ubiquitous as a steering column in automobiles. Its architecture is fairly simple compared to a general over-constrained 6R mechanism which makes it easier to derive the input-output relations. There have been different approaches in the literature to derive its input-output relations proving that it is a constant velocity transmitter when the angle between the input shaft and the central yoke is equal to the angle between the central yoke and the output shaft [11], [12], [13]. The constant-velocity transmission property of a double-Hooke's joint is exploited in this paper to reconfigure the first revolute joint axis in each limb of a 4-RUU PM.

The organization of the paper is as follows: Section II presents the architecture of the dual reconfigurable 4-rRUU PM along with the architecture of its constituent doubleHooke's joint linkage. Section III deals with the derivation of constraint equations and the determination of operation modes of 4rRUU PMs for some specific orientations of the base and revolute joint axes. Section IV concludes the paper and puts forth some open issues associated with the construction of a functional 4-rRUU PM prototype.

\section{THE 4-rRUU PARALLEL MANIPULATOR}

\section{A. Manipulator Architecture}

The architecture of the dual reconfigurable 4-rRUU PM with a square base and a platform is shown in Fig. 1 and its constituent double-Hooke's joint linkage is shown in Fig. 2.

A reconfigurable revolute joint (rR) and two universal joints (UU) mounted in series constitute each limb of the 4-rRUU PM. Point $L_{i}, i=1,2,3,4$ lies on the pivotal axis of the double-Hooke's joint linkage as shown in Fig. 2. Point $A_{i}$ lies on the first revolute joint axis of the 4-rRUU PM and it can be obtained from point $L_{i}$ by traversing a horizontal distance of $l_{i}$ along the first revolute joint axis. Points $B_{i}$ and $C_{i}$ are the geometric centers of the first and the second universal joints, respectively. Points $L_{i}$ and $C_{i}$ form the corners of the square base and the platform, respectively. $\mathscr{F}_{O}$ and $\mathscr{F}_{P}$ are the coordinate frames attached to the fixed base and the moving platform such that their origins $O$ and $P$ lie at the centers of the respective squares. The revolute-joint axes vectors in $i$-th limb are marked $\mathbf{s}_{i j}, i=1,2,3,4 ; j=1, \ldots, 5$. Vectors $\mathbf{s}_{i 1}$ and $\mathbf{s}_{i 2}$ are 


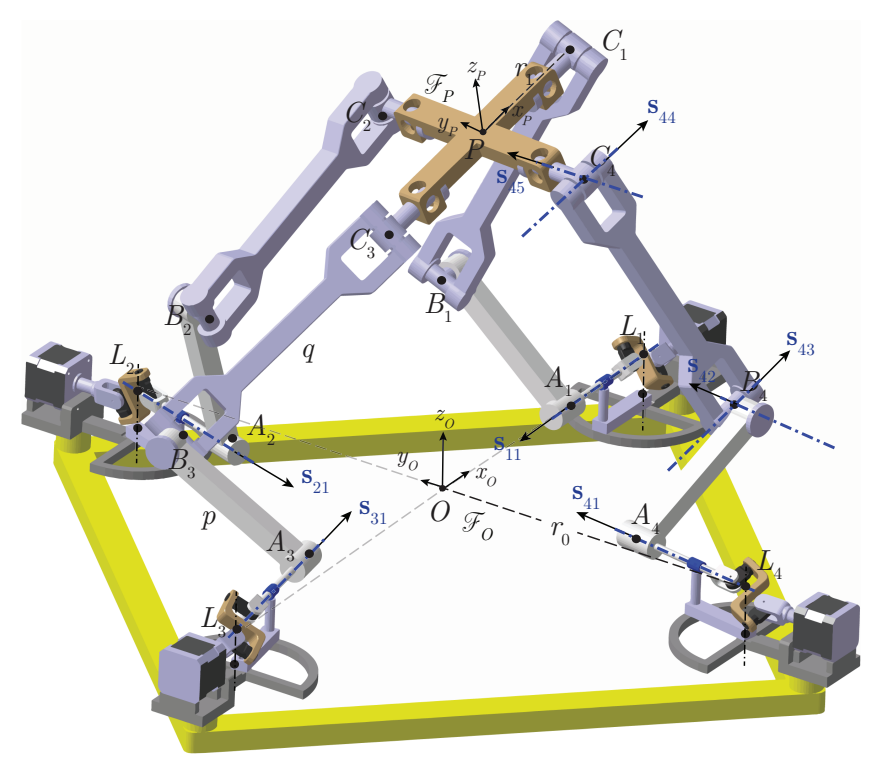

Fig. 1: A 4-rRUU parallel manipulator

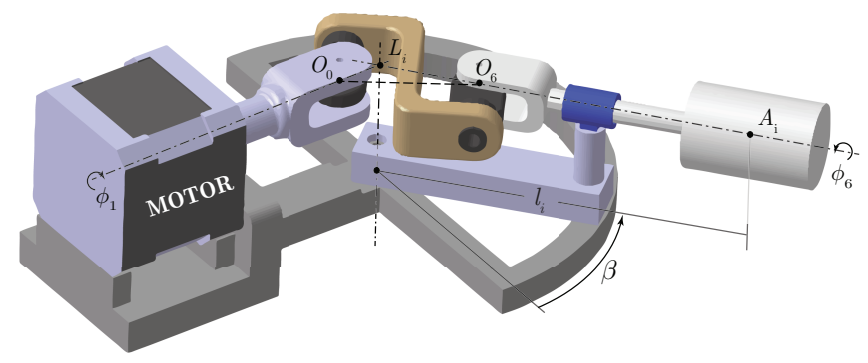

Fig. 2: Double Hooke's joint

always parallel, so are vectors $\mathbf{s}_{i 3}$ and $\mathbf{s}_{i 4}$. For simplicity, it is assumed that the orientation of vector $\mathbf{s}_{i 1}$ expressed in coordinate frame $\mathscr{F}_{O}$ is the same as that of $\mathbf{s}_{i 5}$ expressed in coordinate frame $\mathscr{F}_{P}$. The position vectors of points $L_{i}, A_{i}, B_{i}$ and $C_{i}$ expressed in frame $\mathcal{F}_{k}, k \in O, P$ are denoted as ${ }^{k} \mathbf{l}_{i},{ }^{k} \mathbf{a}_{i},{ }^{k} \mathbf{b}_{i}$ and ${ }^{k} \mathbf{c}_{i}$, respectively. $r_{0}$ and $r_{1}$ are half the diagonals of the base and the moving platform squares, respectively. $p$ and $q$ are the link lengths.

\section{B. Double-Hooke's joint linkage}

The double Hooke's joint linkage is shown in Fig. 2. The first three and the last three revolute joint axes intersect at points $O_{0}$ and $O_{6}$, respectively. The first revolute joint is driven by a motor with an input angle of $\phi_{1}$ and the last revolute joint rotates with an output angle of $\phi_{6}$ and their axes intersect at point $L_{i}, i=1,2,3,4$. It is noteworthy that for a constant-velocity transmission, the triangle $\triangle O_{0} O_{6} L_{i}$ must be isosceles with $O_{0} L_{i}=O_{6} L_{i}$. The angle between the input and the output shafts is denoted as $\beta \in[0, \pi]$. Since the double-Hooke's joint is known to be a constantvelocity transmitter, the following input-output relationship holds [11], [12], [13]:

$$
\phi_{6}=-\phi_{1}
$$

Figure 3 shows the top view of the 4-rRUU PM without the links. For architectural reconfigurability, the reconfigurable

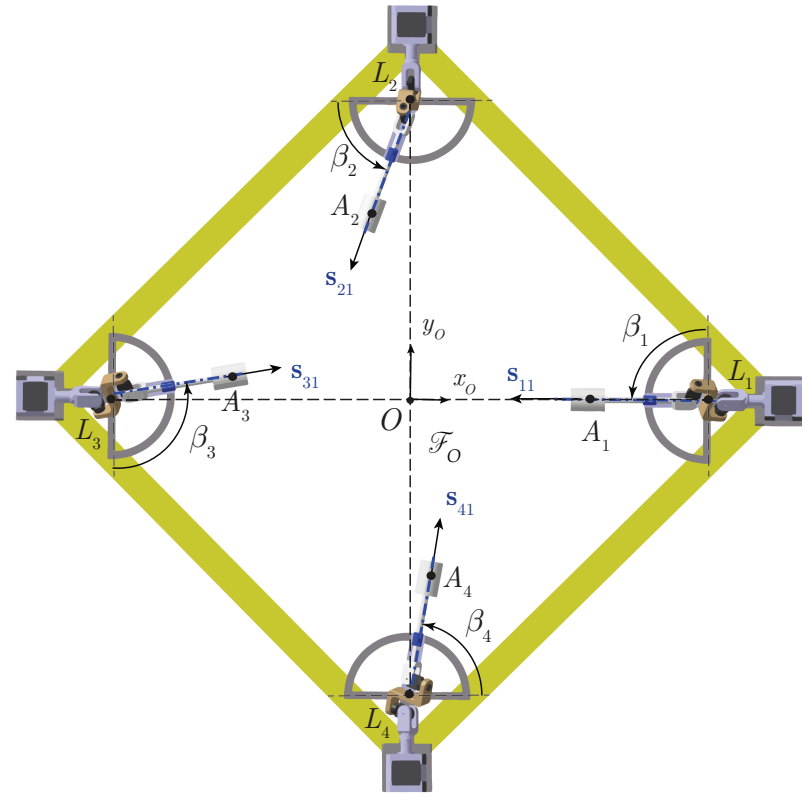

Fig. 3: Possible orientations of the base revolute joint

revolute joint axis in the base is allowed to have a horizontal orientation $\beta_{i}, i=1,2,3,4$. It is noteworthy that $\beta_{i}$ will be changed manually in the prototype under construction.

\section{Operation Mode Analysis}

\section{A. Constraint Equations}

Since the reconfigurable revolute joint is actuated, a RUU limb must satisfy the following two constraints:

1) The second revolute joint axis, the fifth revolute joint axis and link $B C$ must lie in the same plane. In other words, the scalar triple product of the corresponding vectors must be null:

$$
g_{i}:\left(\mathbf{b}_{i}-\mathbf{c}_{i}\right)^{T}\left(\mathbf{s}_{i 2} \times \mathbf{s}_{i 5}\right)=0, \quad i=1,2,3,4
$$

2) The length of link $B C$ must be $q$ :

$$
g_{i+4}:\left\|\mathbf{b}_{i}-\mathbf{c}_{i}\right\|-q=0, \quad i=1,2,3,4
$$

Since the length of link $B C$ does not affect the operation modes of the 4-rRUU PM, only the principal geometric constraint from Eq. (2) is considered. To express it algebraically, the homogeneous coordinates of the necessary vectors are listed below:

$$
\begin{aligned}
& { }^{0} \mathbf{l}_{i}=\mathbf{R}_{z}\left(\lambda_{i}\right)\left[1, r_{0}, 0,0\right]^{T} \\
& { }^{0} \mathbf{a}_{i}={ }^{0} \mathbf{l}_{i}+\mathbf{R}_{z}\left(\lambda_{i}+\beta_{i}\right)\left[0,0, l_{i}, 0\right]^{T} \\
& { }^{0} \mathbf{b}_{i}={ }^{0} \mathbf{a}_{i}+\mathbf{R}_{z}\left(\lambda_{i}+\beta_{i}\right)\left[0, p \cos \left(\theta_{i}\right), 0, p \sin \left(\theta_{i}\right)\right]^{T} \\
& { }^{0} \mathbf{c}_{i}=\mathbf{F ~} \mathbf{R}_{z}\left(\lambda_{i}\right)\left[1, r_{1}, 0,0\right]^{T} \\
& { }^{0} \mathbf{s}_{i 2}=\mathbf{R}_{z}\left(\lambda_{i}+\beta_{i}\right)[0,0,1,0]^{T}, \\
& { }^{0} \mathbf{s}_{i 5}=\mathbf{F} \mathbf{R}_{z}\left(\lambda_{i}+\beta_{i}\right)[0,0,1,0]^{T}, \quad i=1,2,3,4 .
\end{aligned}
$$

where $\mathbf{R}_{z}(\cdot)$ is the homogeneous rotation matrix about the $z$ axis, $\lambda_{i}$ for the $i$-th limb is given by $\lambda_{1}=0, \lambda_{2}=\frac{\pi}{2}, \lambda_{3}=$ $\pi, \lambda_{4}=\frac{3 \pi}{2}$ and $\theta_{i}$ is the actuated joint angle. $\mathbf{F}$ is the 
transformation matrix consisting of Study parameters $x_{j}$ and $y_{j}, j=0,1,2,3$ :

$$
\begin{aligned}
& \mathbf{F}=\frac{1}{\Delta}\left[\begin{array}{cccc}
\Delta & 0 & 0 & 0 \\
d_{1} & r_{11} & r_{12} & r_{13} \\
d_{2} & r_{21} & r_{22} & r_{23} \\
d_{3} & r_{31} & r_{32} & r_{33}
\end{array}\right] \\
& \text { with } \quad \Delta=x_{0}^{2}+x_{1}^{2}+x_{2}^{2}+x_{3}^{2} \neq 0 \\
& \text { and } \quad r_{11}=x_{0}^{2}+x_{1}^{2}-x_{2}^{2}-x_{3}^{2} \\
& r_{12}=-2 x_{0} x_{3}+2 x_{1} x_{2} \\
& r_{13}=2 x_{0} x_{2}+2 x_{1} x_{3} \\
& r_{21}=2 x_{0} x_{3}+2 x_{1} x_{2} \\
& r_{22}=x_{0}{ }^{2}-x_{1}{ }^{2}+x_{2}{ }^{2}-x_{3}^{2} \\
& r_{23}=-2 x_{0} x_{1}+2 x_{2} x_{3} \\
& r_{31}=-2 x_{0} x_{2}+2 x_{1} x_{3} \\
& r_{32}=2 x_{0} x_{1}+2 x_{2} x_{3} \\
& r_{33}=x_{0}{ }^{2}-x_{1}{ }^{2}-x_{2}{ }^{2}+x_{3}^{2} \\
& d_{1}=-2 x_{0} y_{1}+2 x_{1} y_{0}-2 x_{2} y_{3}+2 x_{3} y_{2}, \\
& d_{2}=-2 x_{0} y_{2}+2 x_{1} y_{3}+2 x_{2} y_{0}-2 x_{3} y_{1}, \\
& d_{3}=-2 x_{0} y_{3}-2 x_{1} y_{2}+2 x_{2} y_{1}+2 x_{3} y_{0} .
\end{aligned}
$$

Thus, Eq. (2) is derived for each limb algebraically by substituting $t_{i}=\tan \left(\frac{\theta_{i}}{2}\right)$ and $w_{i}=\tan \left(\frac{\beta_{i}}{2}\right), i=1,2,3,4$. The constraint polynomials $g_{i}, i=1,2,3,4$ form the following ideal $^{1}$ :

$$
\mathcal{I}=\left\langle g_{1}, g_{2}, g_{3}, g_{4}\right\rangle \subseteq k\left[x_{0}, x_{1}, x_{2}, x_{3}, y_{0}, y_{1}, y_{2}, y_{3}\right]
$$

To simplify the determination of the operation modes, the 4rRUU PM is split into two 2-rRUU PMs [8] by considering two ideals:

$$
\begin{aligned}
\mathcal{I}_{(I)} & =\left\langle g_{1}, g_{3}\right\rangle \\
\mathcal{I}_{(I I)} & =\left\langle g_{2}, g_{4}\right\rangle
\end{aligned}
$$

Even after substituting the design parameters, it was impossible to calculate the primary decomposition of ideals $\mathcal{I}_{(I)}$ and $\mathcal{I}_{(I I)}$ for a general $\beta_{i}$ and it remains an open issue. Consequently, some special configurations of the 4-rRUU $\mathrm{PM}$ are considered and their operation modes are determined as follows:

\section{B. Operation Modes of some Specific 4-RUU PMs}

1) $\beta_{1}=\beta_{2}=\beta_{3}=\beta_{4}=\frac{\pi}{2}$ : For the PM shown in Fig. 4 , the constraint equations are derived from Eqs. (2) and (4) as

\footnotetext{
${ }^{1}$ The ideal generated by the given polynomials is the set of all combinations of these polynomials using coefficients from the polynomial ring $k\left[x_{0}, x_{1}, x_{2}, x_{3}, y_{0}, y_{1}, y_{2}, y_{3}\right]$ [14].
}

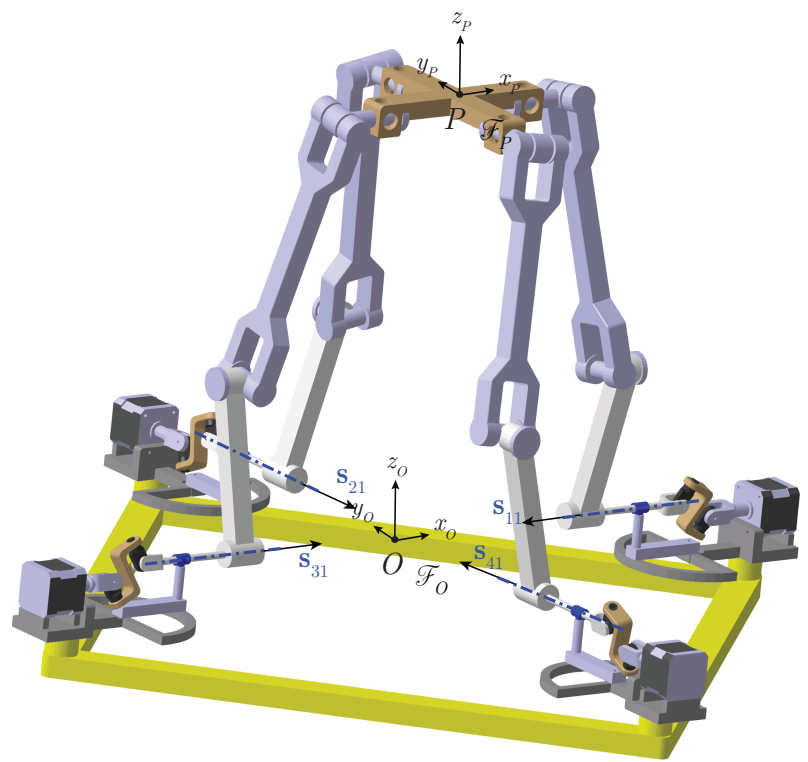

Fig. 4: A 4-rRUU PM with horizontal and intersecting base revolute joint axes

follows:

$$
\begin{aligned}
g_{1}:= & \left(-p t_{1}^{2}+p\right) x_{0} x_{2}+2 p t_{1} x_{0} x_{3}+2 p t_{1} x_{1} x_{2} \\
& +\left(p t_{1}^{2}-p\right) x_{1} x_{3}+\left(2 t_{1}^{2}+2\right) x_{2} y_{2}+\left(2 t_{1}{ }^{2}\right. \\
& +2) x_{3} y_{3}=0 \\
g_{2}:= & \left(-p t_{2}{ }^{2}+r_{0} t_{2}{ }^{2}-r_{1} t_{2}{ }^{2}+p+r_{0}-r_{1}\right) x_{0} x_{2} \\
& +2 t_{2} p x_{0} x_{3}+2 t_{2} p x_{1} x_{2}+\left(p t_{2}{ }^{2}-r_{0} t_{2}{ }^{2}-r_{1} t_{2}{ }^{2}\right. \\
& \left.-p-r_{0}-r_{1}\right) x_{1} x_{3}+\left(2 t_{2}{ }^{2}+2\right) x_{2} y_{2}+\left(2 t_{2}{ }^{2}\right. \\
& +2) x_{3} y_{3}=0 \\
g_{3}:= & \left(-p t_{3}{ }^{2}+p\right) x_{0} x_{2}+2 p t_{3} x_{0} x_{3}+2 p t_{3} x_{1} x_{2} \\
& +\left(p t_{3}{ }^{2}-p\right) x_{1} x_{3}+\left(2 t_{3}{ }^{2}+2\right) x_{2} y_{2}+\left(2 t_{3}{ }^{2}\right. \\
& +2) x_{3} y_{3}=0 \\
g_{4}:= & \left(-p t_{4}{ }^{2}-r_{0} t_{4}{ }^{2}+r_{1} t_{4}{ }^{2}+p-r_{0}+r_{1}\right) x_{0} x_{2} \\
& +2 t_{4} p x_{0} x_{3}+2 t_{4} p x_{1} x_{2}+\left(p t_{4}{ }^{2}+r_{0} t_{4}{ }^{2}+r_{1} t_{4}{ }^{2}\right. \\
& \left.-p+r_{0}+r_{1}\right) x_{1} x_{3}+\left(2 t_{4}{ }^{2}+2\right) x_{2} y_{2}+\left(2 t_{4}{ }^{2}\right. \\
& +2) x_{3} y_{3}=0
\end{aligned}
$$

The primary decomposition of ideals $I_{(I)}$ and $I_{(I I)}$ shown in Eq. (7) leads to three sub-ideals each. Among them, the third sub-ideals $\mathcal{I}_{3(I)}$ and $\mathcal{I}_{3(I I)}$ correspond to a mixed mode and are of little importance in this context. The other two sub-ideals $\mathcal{I}_{k(I)}$ and $\mathcal{I}_{k(I I)}, k=1,2$ are as follows:

$$
\begin{gathered}
\mathcal{I}_{(I)}=\mathcal{I}_{1(I)} \cap \mathcal{I}_{2(I)} \cap \mathcal{I}_{3(I)}, \\
\text { where } \mathcal{I}_{1(I)}=\left\langle x_{0}, x_{1}, x_{2} y_{2}+x_{3} y_{3}\right\rangle \\
\mathcal{I}_{2(I)}=\left\langle x_{2}, x_{3}, x_{0} y_{0}+x_{1} y_{1}\right\rangle \\
\mathcal{I}_{(I I)}=\mathcal{I}_{1(I I)} \cap \mathcal{I}_{2(I I)} \cap \mathcal{I}_{3(I I)}, \\
\text { where } \mathcal{I}_{1(I I)}=\left\langle x_{0}, x_{1}, x_{2} y_{2}+x_{3} y_{3}\right\rangle \\
\mathcal{I}_{2(I I)}=\left\langle x_{2}, x_{3}, x_{0} y_{0}+x_{1} y_{1}\right\rangle
\end{gathered}
$$

As a result, the first two operation modes of the 4-rRUU PM 
shown in Fig. 4 are:

$$
\begin{aligned}
& \mathcal{I}_{1}=\mathcal{I}_{1(I)} \cup \mathcal{I}_{1(I I)}=\left\langle x_{0}, x_{1}, x_{2} y_{2}+x_{3} y_{3}\right\rangle \\
& \mathcal{I}_{2}=\mathcal{I}_{2(I)} \cup \mathcal{I}_{2(I I)}=\left\langle x_{2}, x_{3}, x_{0} y_{0}+x_{1} y_{1}\right\rangle
\end{aligned}
$$

Substituting the condition $x_{0}=x_{1}=x_{2} y_{2}+x_{3} y_{3}=0$ in the transformation matrix in Eq. (5) yields:

$$
\mathbf{F}_{1}=\left[\begin{array}{cccc}
1 & 0 & 0 & 0 \\
-\frac{2 y_{3}}{x_{2}} & -1 & 0 & 0 \\
2\left(x_{2} y_{0}-x_{3} y_{1}\right) & 0 & x_{2}^{2}-x_{3}^{2} & 2 x_{2} x_{3} \\
2\left(x_{2} y_{1}+x_{3} y_{0}\right) & 0 & 2 x_{2} x_{3} & -x_{2}^{2}+x_{3}^{2}
\end{array}\right]
$$

From the transformation matrix, it can be observed that the operation mode is a 4-dof Schönflies mode in which the translational motions are parametrized by $y_{0}, y_{1}$ and $y_{3}$ and the rotational motion is parametrized by $x_{2}, x_{3}$ and $x_{2}^{2}+x_{3}^{2}=1$. In this operation mode, the platform is upside down with the $z_{P}$-axis pointing in a direction opposite to the $z_{O}$-axis. The rotational motion is about $x_{O}$-axis.

Similarly, substituting the condition $x_{0}=x_{1}=x_{2} y_{2}+$ $x_{3} y_{3}=0$ in the transformation matrix in Eq. (5) yields:

$\mathbf{F}_{2}=\left[\begin{array}{cccc}1 & 0 & 0 & 0 \\ -\frac{2 y_{1}}{x_{0}} & 1 & 0 & 0 \\ -2\left(x_{0} y_{2}-x_{1} y_{3}\right) & 0 & x_{0}{ }^{2}-x_{1}{ }^{2} & -2 x_{0} x_{1} \\ -2\left(x_{0} y_{3}+2 x_{1} y_{2}\right) & 0 & 2 x_{0} x_{1} & x_{0}{ }^{2}-x_{1}{ }^{2}\end{array}\right]$

In this case, the operation mode is also a 4-dof Schönflies mode in which the translational motions are parametrized by $y_{1}, y_{2}$ and $y_{3}$ and the rotational motion is parametrized by $x_{0}, x_{1}$ and $x_{0}^{2}+x_{1}^{2}=1$. The platform is in upright position with rotational motion about $x_{O}$-axis.

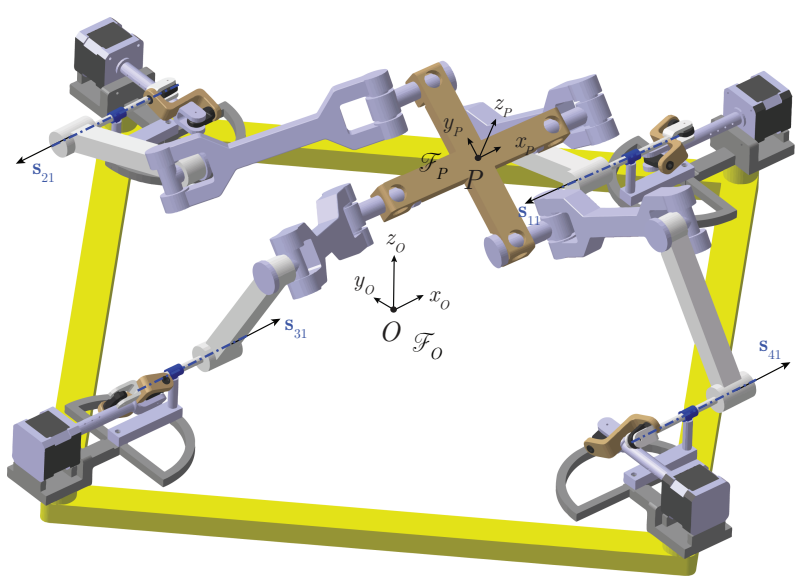

Fig. 5: A 4-RUU PM with horizontal and parallel base revolute joint axes

2) $\beta_{1}=\beta_{3}=\frac{\pi}{2}, \beta_{3}=\beta_{4}=0$ : For the PM shown in Fig. 5 with $w_{1}=w_{3}=1$ and $w_{2}=w_{4}=0$, the constraint equations are derived according to Eqs. (2) and (4) as follows:

$$
\begin{aligned}
g_{1}:= & \left(-p t_{1}^{2}+p\right) x_{0} x_{2}+2 p t_{1} x_{0} x_{3}+2 p t_{1} x_{1} x_{2} \\
& +\left(p t_{1}{ }^{2}-p\right) x_{1} x_{3}+\left(2{t_{1}}^{2}+2\right) x_{2} y_{2}+\left(2 t_{1}{ }^{2}\right. \\
& +2) x_{3} y_{3}=0 \\
g_{2}:= & \left(-p t_{2}{ }^{2}+p\right) x_{0} x_{1}+2 p t_{2} x_{0} x_{3}-2 p t_{2} x_{1} x_{2} \\
& +\left(-p t_{2}{ }^{2}+p\right) x_{2} x_{3}+\left(2 t_{2}{ }^{2}+2\right) x_{1} y_{1}+\left(2 t_{2}{ }^{2}\right. \\
& +2) x_{3} y_{3}=0 \\
g_{3}:= & \left(-p t_{3}{ }^{2}+p\right) x_{0} x_{2}+2 p t_{3} x_{0} x_{3}+2 p t_{3} x_{1} x_{2} \\
& +\left(p t_{3}{ }^{2}-p\right) x_{1} x_{3}+\left(2 t_{3}{ }^{2}+2\right) x_{2} y_{2}+\left(2 t_{3}{ }^{2}\right. \\
& +2) x_{3} y_{3}=0 \\
g_{4}:= & \left(-p t_{4}{ }^{2}+p\right) x_{0} x_{1}+2 p t_{4} x_{0} x_{3}-2 p t_{4} x_{1} x_{2} \\
& +\left(-p t_{4}{ }^{2}+p\right) x_{2} x_{3}+\left(2 t_{4}{ }^{2}+2\right) x_{1} y_{1}+\left(2 t_{4}{ }^{2}\right. \\
& +2) x_{3} y_{3}=0 \\
&
\end{aligned}
$$

The primary decomposition of ideals $I_{(I)}$ and $I_{(I I)}$ shown in Eq. (7) leads to three sub-ideals each, of which the two sub-ideals $\mathcal{I}_{k(I)}$ and $\mathcal{I}_{k(I I)}, k=1,2$ are as follows:

$$
\begin{gathered}
\mathcal{I}_{(I)}=\mathcal{I}_{1(I)} \cap \mathcal{I}_{2(I)} \cap \mathcal{I}_{3(I)}, \\
\text { where } \mathcal{I}_{1(I)}=\left\langle x_{0}, x_{1}, x_{2} y_{2}+x_{3} y_{3}\right\rangle \\
\mathcal{I}_{2(I)}=\left\langle x_{2}, x_{3}, x_{0} y_{0}+x_{1} y_{1}\right\rangle \\
\mathcal{I}_{(I I)}=\mathcal{I}_{1(I I)} \cap \mathcal{I}_{2(I I)} \cap \mathcal{I}_{3(I I)}, \\
\text { where } \mathcal{I}_{1(I I)}=\left\langle x_{0}, x_{2}, x_{1} y_{1}+x_{3} y_{3}\right\rangle \\
\mathcal{I}_{2(I I)}=\left\langle x_{1}, x_{3}, x_{0} y_{0}+x_{2} y_{2}\right\rangle
\end{gathered}
$$

As a result, the first two operation modes of the 4-RUU PM are:

$$
\begin{aligned}
& \mathcal{I}_{1}=\mathcal{I}_{1(I)} \cup \mathcal{I}_{1(I I)}=\left\langle x_{0}, x_{1}, x_{2}, y_{3}\right\rangle \\
& \mathcal{I}_{2}=\mathcal{I}_{2(I)} \cup \mathcal{I}_{2(I I)}=\left\langle x_{1}, x_{2}, x_{3}, y_{0}\right\rangle
\end{aligned}
$$

Substituting the condition $x_{0}=x_{1}=x_{2}=y_{3}=0$ in the transformation matrix in Eq. (5) yields:

$$
\mathbf{F}_{1}=\left[\begin{array}{cccc}
1 & 0 & 0 & 0 \\
\frac{2 y_{2}}{x_{3}} & -1 & 0 & 0 \\
-\frac{2 y_{1}}{x_{3}} & 0 & -1 & 0 \\
\frac{2 y_{0}}{x_{3}} & 0 & 0 & 1
\end{array}\right]
$$

From the transformation matrix, it can be deduced that the operation mode is a 3-dof pure translational mode parametrized by $y_{0}, y_{1}$ and $y_{2}$ when $x_{3}=1$. In this operation mode, the platform is upside down with the $z_{P}$-axis pointing downwards.

Similarly, substituting the condition $x_{1}=x_{2}=x_{3}=$ 
$y_{0}=0$ in the transformation matrix in Eq. (5) yields:

$$
\mathbf{F}_{2}=\left[\begin{array}{cccc}
1 & 0 & 0 & 0 \\
-\frac{2 y_{1}}{x_{0}} & 1 & 0 & 0 \\
-\frac{2 y_{2}}{x_{0}} & 0 & 1 & 0 \\
-\frac{2 y_{3}}{x_{0}} & 0 & 0 & 1
\end{array}\right]
$$

In this case, the operation mode is also a 3-dof translational mode parametrized by $y_{1}, y_{2}$ and $y_{3}$ when $x_{0}=1$. Since the rotation matrix is identity, the platform is in upright position with $z_{P}$-axis pointing upwards.

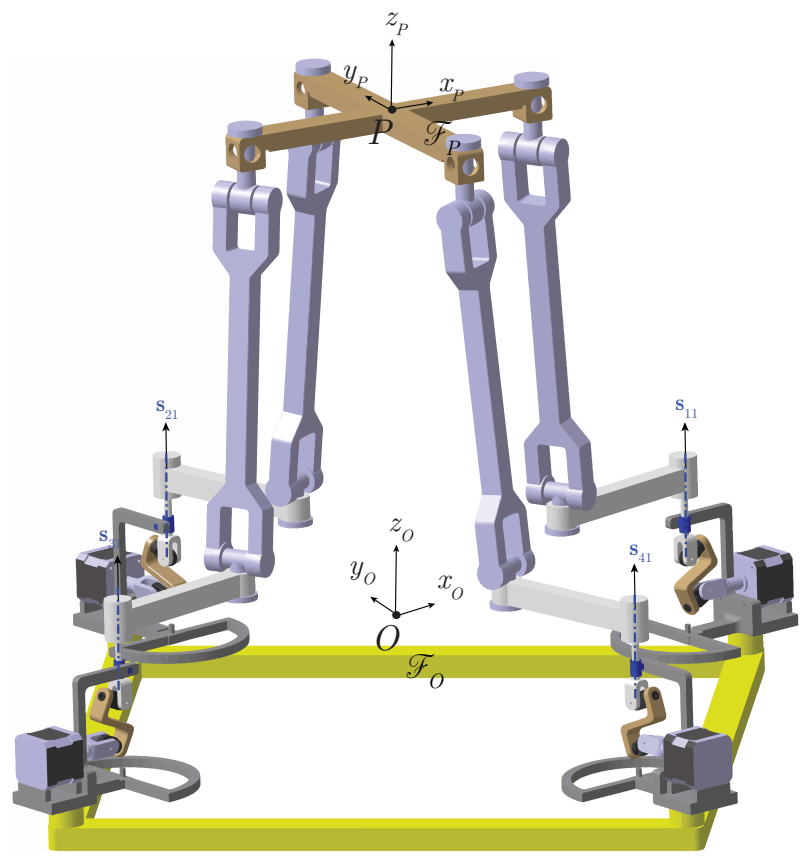

Fig. 6: A dual reconfigurable 4-rRUU PM with vertical base revolute joint axes

3) Vertical base and platform revolute joint axes: The double-Hooke's joint allows a planar transmission and hence the 4-rRUU PM can have any orientation of the base revolute joints such that $\beta_{i} \in[0, \pi]$. Additionally, with the help of a L-fixture, it is possible to have a vertical orientation of the base revolute joint axes as shown in Fig. 6 .

Reconfiguration analysis of this mechanism already exists in the literature [8], where it was shown to have three operation modes. The first operation mode is a 4-dof Schönflies mode in which the platform is upside down and the rotational axis is parallel to $z_{O}$-axis. The second operation mode is a 4-dof Schönflies mode with the rotational axis parallel to $z_{O^{-}}$ axis, but in this case, the posture of the platform is upright. The third operation mode is a 2-dof coupled motion mode and is less relevant from a practical view point.

\section{CONCLUSIONS AND FUTURE WORK}

A dual reconfigurable 4-rRUU PM consisting of a reconfigurable revolute joint based on the double-Hooke's joint linkage was presented in this paper. It was shown how a double-Hooke's joint linkage can be exploited to impart an architectural reconfigurability to a 4-RUU PM. The resulting dual reconfigurable 4-rRUU PM was shown to exhibit at least the following operation modes: a pure translational operation mode and Schönflies motion modes with different axes of rotation depending on the orientation of base revolute joint axes.

As a part of the future work, the operation modes will be determined as a function of the angle $\beta$ which will assist in recognizing all possible platform motion types of the 4-rRUU PM for $\beta \in[0, \pi]$. Furthermore, a detailed design of the 4-rRUU PM will be performed in order to construct a functional prototype exhibiting different types of reconfigurability. It should be noted that in the prototype, the orientation of the revolute joint axes in the moving platform will have to be changed manually and the choice of a joint to have all planar orientations is still an open issue.

\section{ACKNOWLEDGMENT}

This work was conducted with the support of both Centrale Nantes and the French National Research Agency (ANR project "Kapamat" \#ANR-14-CE34-0008-01). We would also like to show our gratitude to Rakesh Shantappa, a former Master's student at Centrale Nantes for his help with the CAD models.

\section{REFERENCES}

[1] D. Zlatanov, I. A. Bonev, and C. M. Gosselin, Constraint Singularities as C-Space Singularities. Dordrecht: Springer Netherlands, 2002, pp. 183-192.

[2] D. R. Walter, M. L. Husty, and M. Pfurner, "A complete kinematic analysis of the SNU 3-UPU parallel robot," Contemporary Mathematics, vol. 496, p. 331, 2009.

[3] D. R. Walter and M. L. Husty, "Kinematic analysis of the TSAI 3-UPU parallel manipulator using algebraic methods," in The 13th IFToMMWorld Congress in Mechanism and Machine Science, Guanajuato, Mexico, 2011, pp. 1-10.

[4] J. Schadlbauer, D. Walter, M. Husty, J. Schadlbauer, D. Walter, and M. Husty, "The 3-rps parallel manipulator from an algebraic viewpoint," Mechanism and Machine Theory, vol. 75, no. Complete, pp. 161-176, 2014.

[5] L. Nurahmi, S. Caro, and P. Wenger, "Operation modes and singularities of 3-PRS parallel manipulators with different arrangements of p-joints," in ASME 2015 International Design Engineering Technical Conferences and Computers and Information in Engineering Conference. American Society of Mechanical Engineers, 2015.

[6] A. Nayak, L. Nurahmi, P. Wenger, and S. Caro, Comparison of 3-RPS and 3-SPR Parallel Manipulators Based on Their Maximum Inscribed Singularity-Free Circle. Cham: Springer International Publishing, 2017, pp. 121-130.

[7] X. Kong, "Reconfiguration analysis of a 3-DOF parallel mechanism using euler parameter quaternions and algebraic geometry method," Mechanism and Machine Theory, vol. 74, pp. 188 - 201, 2014.

[8] L. Nurahmi, S. Caro, P. Wenger, J. Schadlbauer, and M. Husty, "Reconfiguration analysis of a 4-RUU parallel manipulator," Mechanism and Machine Theory, vol. 96, pp. 269-289, 2016.

[9] R. A. Srivatsan and S. Bandyopadhyay, "On the position kinematic analysis of mapaman: a reconfigurable three-degrees-of-freedom spatial parallel manipulator," Mechanism and Machine Theory, vol. 62, pp. $150-165,2013$.

[10] D. Gan, J. S. Dai, J. Dias, and L. D. Seneviratne, "Optimal design of a metamorphic parallel mechanism with reconfigurable 1T2R and 3R motion based on unified motion/force transmissibility," $\mathrm{p}$. V05BT07A045, 2016. 
[11] J. Baker, "Displacementclosure equations of the unspecialised doublehooke's-joint linkage," Mechanism and Machine Theory, vol. 37, no. 10 , pp. 1127 - 1144, 2002.

[12] P. Dietmaier, "Simply overconstrained mechanisms with rotational joints," Habilitation thesis, Graz University of Technology, 1995.

[13] C. Mavroidis and B. Roth, "Analysis of overconstrained mechanisms," Journal of Mechanical Design, vol. 117, no. 1, pp. 69-74, Mar 1995.

[14] D. A. Cox, J. Little, and D. O'Shea, Ideals, Varieties, and Algorithms: An Introduction to Computational Algebraic Geometry and Commutative Algebra, 3/e (Undergraduate Texts in Mathematics). Secaucus, NJ, USA: Springer-Verlag New York, Inc., 2007. 